Instituto Internacional de Investigación y Desarrollo Tecnológico Educativo INDTEC, C.A.

DOI: https://doi.org/10.29394/scientific.issn.2542-2987.2017.0.0.21.394-410

OAI-PMH: http://www.indteca.com/ojs/index.php/Revista Scientific/oai

\title{
Valores Éticos del Gerente Universitario en los Contextos de la Postmodernidad
}

Autora: Sonia María Aguilar Rondón Universidad Nacional Experimental "Rafael María Baralt", UNERMB sonia aguilar1965@hotmail.com

Trujillo, Venezuela

\section{Resumen}

El artículo está centrado en naturalezas dominantes de las organizaciones posmodernas en el siglo XXI, aunado a los Valores Éticos que cobran cada vez más importancia en cuanto a la consideración de la relación que estos guardan con el desempeño de la gerencia universitaria. Así, el propósito consiste en determinar la importancia que tienen los valores éticos en el gerente universitario en contextos de la posmodernidad. En lo que respecta a la metodología, se consideró aplicar una investigación de tipo documental, con diseño bibliográfico buscando fundamentar las ideas y puntos de vista expresados con base en el análisis de las referencias bibliográficas consultadas de acuerdo con el propósito del trabajo. Las conclusiones resultantes, indican que los valores éticos se conciben como guías para resaltar el comportamiento humano; además, la ética del gerente universitario debe basarse en la misión y visión de las organizaciones posmodernas.

Palabras clave: valores; ética; gerencia; posmodernidad. 


\title{
Ethical Values of the University Manager in the Contexts of Postmodernity
}

\begin{abstract}
The article focuses on dominant natures of postmodern organizations in the 21st century, coupled with ethical values that are increasingly important in terms of their relationship with the performance of university management. Thus, the purpose is to determine the importance of ethical values in the university manager in contexts of postmodernity. Regarding the methodology, it was considered to apply documentary research, with a bibliographic design, seeking to base the ideas and points of view expressed based on the analysis of bibliographical references consulted according to the purpose of the work. The resulting conclusions indicate that ethical values are conceived as guidelines for highlighting human behavior; In addition, the ethics of the university manager should be based on the mission and vision of postmodern organizations.
\end{abstract}

Keywords: values; ethics; management; postmodernity. 


\section{Introducción}

En estos momentos, la educación universitaria está ubicada en el escalafón de mayor relevancia dentro del sistema educativo venezolano. En los últimos años el sistema universitario ha ejercitado transformaciones importantes. Entre ellas, la globalización, internacionalización, así como las nuevas exigencias profesionales, tecnológicas que le han afectado lo que conlleva a que ésta esté en la actualidad, en un momento de cambio y armonía.

Lo cierto, es que la gerencia universitaria venezolana, caso que nos atañe, debe identificarse proactiva y eficiente ante los nuevos retos que desafía, por lo tanto, debe avalar rendimiento, ética, participación, compromiso, manejo conveniente de sus recursos, adjudicarse la cultura de la evaluación, de la calidad y del valor añadido diferencial como bases sobre las que asentar la idoneidad universitaria.

Entretanto, la gerencia educativa, en cuanto a los valores éticos que la deben regir, es motivo de cuestionamiento desde diversos enfoques teóricos por las consecuencias que tiene dentro de los contextos de la educación venezolana. Por lo tanto, la gerencia en el ámbito universitario constituye el soporte estructural de la gestión que afecta a las organizaciones; una ordenación cada vez más profesionalizada y motivada que actúa en un entorno cada día más exigente en términos de exigir calidad y oportunidad.

De esto se infiere, que el gerente universitario nunca debe despojarse de los valores éticos que alimentan, guían y dan sentido a su ejercicio profesional. A la luz de las ideas anteriores, ejercer la ética es uno de los argumentos más urgentes que atender para mejorar la calidad de la experiencia profesional en la actualidad. En un momento, en que nuestras organizaciones empresariales, organismos públicos están inmersos en la búsqueda de opciones, aprobaciones para lograr competitividad, afianzamiento económico y credibilidad social, son importantes los esfuerzos que ayuden a la creación de 
una conciencia colectiva en torno a los valores éticos de la probidad como forma genuina de lograr resultados positivos en cualquier aspecto de la vida. Como complemento de la temática, Bello, (2008) establece en relación a las acciones para construir la ética:

Algunos aspectos, se pueden constituir en antivalores que, en algún momento, se disfrazaron de virtudes en el carnaval de la vida. Las debilidades, por su parte, indicaran oportunidades para reforzar aspectos descuidados de nuestra personalidad... Así que vamos desde el reconocimiento de nuestras fortalezas y debilidades en las acciones cotidianas hacia la identificación del valor, haciendo una abstracción, y luego, desde esta conciencia del valor hacia la práctica del mismo en los pequeños actos del día a día. (pág. 57).

Dicho de otro modo, la persona que se siente bien consigo misma y tiene plena confianza en su poder personal para lograr las cosas que se merece, hace un esfuerzo sensato para superar las presiones internas y externas que lo invitan a hacer lo que siente que es erróneo, tiene el carácter para esperar su tiempo, para hacer lo correcto oportunamente con el propósito de obtener sus beneficios.

Ahora bien, desde la figura de la gerencia universitaria son muchas las variables que pueden someterse a estudio porque sin duda, permitirían conocer cada vez más la dinámica que debe predominar en este gerente como modelo a seguir para la organización que regenta. De todas esas variables, en la presente indagación el factor ético constituye un asunto social importante puesto que admite hacer referencia a la ontología del ser humano, como ser social capaz de transformar la realidad en beneficio del bien común. Al respecto, Correa, (2006) refiere: "al gerente universitario hay que entenderlo como un ser complejo; pues sus múltiples relaciones están dadas de tal forma que invitan a resignificar una postura del concepto en torno a la condición humana" (pág. 32). 
De este modo, la incorporación de cuestiones éticas y la modificación o creación de condiciones puede que hagan posible que el contexto de gerencia también sea de aprendizaje ético; ello requerirá, el establecimiento de pautas o nuevo normativo, que en nuestro caso, están descritos en los instrumentos de ley, entre los que cabe mencionar: a juicio de Freites, (2012:3) lo destacado en la primera línea estratégica general del Primer Plan de Desarrollo de la Nación 2007-2013; La Nueva Ética Socialista, "la cual se basa en la refundación ética y moral de la nación, cuyas raíces están en la fusión de los valores y principios de la corriente humanista, del socialismo y de la herencia histórica del pensamiento de Simón Bolívar". Se plantea en el referido plan:

La nueva ética socialista es la superación de la ética del capital para crear conciencia revolucionaria bajo una nueva moral colectiva, que solo puede ser alcanzada mediante la dialéctica de la lucha por la transformación material de la sociedad y el desarrollo de la espiritualidad (pág. 3)

En otras palabras, los valores éticos no son, el resultado de una intuición y mucho menos de una indagación pasiva, ni tampoco de actitudes trasladadas sin significación propia por el sujeto; es más complicado y multilateral que trata los elementos de la personalidad, sus contenidos y sus formas de expresión a través de conductas y procederes. Así, solo se puede gerenciar basado en un marco ético a través de conocimientos, habilidades de valoración reflexión y la actividad práctica.

Al mismo tiempo, los contextos de la posmodernidad, están marcados por un episodio de la crisis de la modernidad, la cual, según Lyon (2000), citado en Useche y Queipo (2005) "se caracteriza por el auge del sector servicio, el rápido cambio tecnológico y las posibilidades que ofrece las telecomunicaciones, convirtiéndose en aspectos decisivos para los intercambios empresariales y sociales" (pág. 280) Es decir, la posmodernidad del siglo XXI pertenece a un período histórico donde la sociedad se 
desenvuelve en base al consumo, la informática, medios de comunicación, así como, la tecnología sofisticada, donde se dan profundas mutaciones para dar respuestas al mercado y situaciones que afectan el ambiente organizacional y natural, la cultura, la calidad, entre otros.

En ese fin, la posmodernidad está adornada de compendios elevados en las organizaciones en el siglo XXI, para lo cual se exploran aspectos bajo la corriente posmodernista sobre los cuales se sustentan éstas para comprender cómo las innovaciones del mercado perturban el desempeño y la direccionalidad organizacional, obligándolas a adecuar y compatibilizar sus propiedades para desafiar las nuevas realidades de caos y complejidad, así como, la utilidad de la tecnología de la información, comunicación, digitalización y aunado a estos elementos la responsabilidad social y la ética son compromisos básicos en la expansión organizacional universitaria.

Con relación en la ética gerencial, Guedez, (2004), refiere "Los actos de las empresas y de los individuos que hacen parte de ellas influyen de manera positiva o negativa a todo lo que está a su entorno. Es por eso que toda empresa tiene códigos de conductas sustentadas en valores" (pág. 69). De este modo, el gerente universitario en los contextos de la posmodernidad deberá ser modelo de ética y compromiso, porque las organizaciones en la postmodernidad demandaran una fuerza laboral capaz de crear, facilitar y aplicar conocimiento, sumamente especializado, capacitado y productivo

Partiendo de las concepciones planteadas y el análisis expuesto emerge la siguiente interrogante: ¿Cuál es la importancia que tienen los valores éticos en el gerente universitario en contextos de la posmodernidad? En ese orden de ideas, se hace pertinente plantear que las organizaciones universitarias, en los momentos vigentes, amplían su función social, porque no solo trabajan por educar a los estudiantes, sino que también buscan incidir en el desarrollo de la familia y la comunidad en que se encuentran situadas, luchan por convertirse en generadoras de compañerismo, seguridad y al 
mismo tiempo, establecen relaciones sociales e institucionales que favorezcan medios interesantes, atractivos en el que se destaque el trabajo en para y con colectivo en un marco de valores éticos compartidos.

Además, este estudio pretende ser fuente de investigación bibliográfica para investigaciones futuras vinculadas con los valores éticos del gerente universitario en contextos posmodernos. En consecuencia, el estudio se considera relevante por ser pertinente con la realidad que se vive en las organizaciones universitarias y por estar concatenado con las líneas políticas del país dirigidas a fortalecer la ética del funcionario académico.

\section{Valores Éticos}

Hablar de Valores Éticos supone referirse a la axiología, que a juicio de Ramos, (2004) la define como "la teoría del valor o de lo que se considera valioso, no solo trata de los valores positivos, sino también de los valores negativos, analizando los principios que permiten considerar que algo es o no valioso, y considerando los fundamentos de tal juicio" (pág. 42-43). Es decir, la investigación de una teoría de los valores ha encontrado una aplicación especial en la ética, ámbito donde el concepto de valor posee una relevancia específica.

Entretanto, la axiología tiene por objeto de estudio la naturaleza o esencia de los valores y de los juicios de valor que puede realizar un individuo; por eso, es muy común y frecuente que a la axiología se la denomine filosofía de valores. Tanto los valores positivos como los negativos son abordados de manera igual por la axiología, mediante el análisis que considera a algo como valioso o no.

En ese orden de ideas, el Consejo Moral Republicano (2006), establece "los valores son principios que marcan la actitud y las conductas de las personas, es decir son aspectos que influyen en su comportamiento y les permite crecer como individuos al entenderlos como aportes positivos al 
desarrollo de sus vidas" (pág. 19). Se infiere entonces, que las cosas no son valiosas por sí mismas, sino que tienen el valor que le damos, por esto cada persona tiene su propia escala de valores. No todos se comportan de igual manera ante las vivencias y problemas de la vida.

Asimismo, Carreras (2001), expone que los valores éticos plantean que "la educación en valores se justifica por la necesidad que tienen los individuos de comprometer a las personas con determinados principios éticos, que sirvan para evaluar las propias acciones y la de los demás" (pág. 101). O lo que es lo mismo, están presentes en la vida frecuente, se revelan mediante conductas y opiniones expresadas oralmente o por escrito, suelen dar lugar a normas sociales. Los valores sirven para "guiar" la conducta de las personas, son el fundamento por el cual se hace o deja de hacer una cosa en un determinado momento.

Al respecto, Rojas (2007) plantea que la ética "debe guiar la profesionalidad de los gerentes universitarios, dentro de un marco de verdadera moralidad y pulcritud, ya que de nada vale un servidor que realice muy bien las funciones encomendadas, si su desempeño no es ético" (pág. 33). En efecto, la ética es el conjunto de recomendaciones que deben mantener los seres humanos asentar en el principio que determina su acción en la sociedad, respaldadas en la ciencia del bien, la sabiduría del ser humano ante las actitudes concretas de su comportamiento.

Cabe destacar, que los valores éticos se han impregnado en el acontecer cotidiano de la gerencia actual, propiciando un retorno a la espiritualidad, la asunción de un comportamiento más integral y esto, según Paz, (2005), "obedece a un signo exclusivamente de emprendedurismo que se relaciona con su fin último, servir a la sociedad donde se inserta" (pág. 56). En tal sentido, es necesario que los gerentes universitarios actúen como agentes de cambio educativo, para lo cual deben ostentar cualidades concretas que respondan a las nuevas reclamaciones humanísticas de la 
educación, requiriéndose para ello un gerente educador con poder moral que exprese sus virtudes al vivir y convivir de acuerdo a una concepción ética de la vida.

\subsection{Gerencia}

La gerencia, concretándola en términos comunes, es una práctica relacionada con la organización de compendios, administración de recursos 0 el logro de objetivos expuestos en una organización. Esta apreciación se apoya en lo expresado por Kurosawa, (2007), para quien gerenciar es "ordenar y utilizar los factores humanos y los recursos organizativos, técnicos 0 financieros, combinándolos para alcanzar los objetivos de las políticas diseñadas" (pág. 25).

De ese modo, practicar la gerencia implica precisamente el desarrollo de ciertas prácticas, experiencias, partiendo del conocimiento de la organización en cuanto a debilidades y fortalezas, así como oportunidades y amenazas, hasta las perspectivas de las personas que completan la misma 0 están relacionadas con ella, pues sólo así, es factible armonizar un trabajo compuesto de todos los elementos institucionales para el logro de los objetivos propuestos.

Además, para George, (2009), "La Ética se considera como una ciencia práctica y normativa que estudia el comportamiento de los hombres, que conviven socialmente bajo una serie de normas que le permiten ordenar sus actuaciones y que el mismo grupo social ha establecido" (pág. 22). No obstante, en este momento, el concepto de la ética en la gerencia abarca un argumento más amplio y dinámico, se relaciona con la gestión institucional, delimitándola como la puesta en práctica de estrategias innovadoras tendentes a obtener un máximo de efectividad en cada uno de los procesos gerenciales cumplidos. Bajo esta perspectiva, se aplica el concepto de gerencia a las organizaciones universitarias, para optimizar la productividad en las mismas. 
En concordancia con lo expresado, un gerente es la persona que planifica, organiza, dirige y controla una organización, con el fin de alcanzar objetivos propuestos, los cuales pueden estar relacionados con patrocinios económicos o determinado servicio social. Atendiendo a estas características, al gerente se le define por su capacidad y destreza para elegir entre varias alternativas la opción correcta, buscando siempre el rendimiento institucional, y entendiendo ésta como el logro de máximos beneficios a partir de un uso estratégico de los recursos existentes. Dicho en otras palabras: saber aprovechar los recursos disponibles en función de los objetivos propuestos, tomando en cuenta las fortalezas y debilidades de la organización; lo cual no inhibe la posibilidad de reorientación de políticas o estrategias de acción para garantizar el éxito y alcanzar la productividad.

Ahora bien, ubicándose en el contexto que corresponde al presente artículo es decir, el ámbito universitario, es posible encontrar una postura heurística de parte de González, (2009) quien afirma: "La gerencia en el campo universitario es el cargo que ocupa el director, jefe, supervisor, quien coordina los recursos a través de planteamientos y organización de acuerdo a lo establecido en la teoría tayloriana" (pág. 45) Podría decirse entonces, que es el cargo de mayor jerarquía o autoridad en una organización, y que es llevado a cabo por un director, el cual tiene como función representar a la misma, tanto internamente, coordinando los recursos, a través de múltiples procesos, como externamente ante la sociedad, donde este viene a ser la fachada principal, es decir la imagen de la organización.

\subsubsection{Gerencia Universitaria}

En las universidades la ética representa un mecanismo indispensable, el cual debe hallarse siempre presente para así garantizar en cierto modo, el éxito de la gestión universitaria, cada vez que se prepara un nuevo periodo académico se deben tomar un conjunto de disposiciones que aumentan la 
satisfacción en el cumplimiento de los objetivos, pero debido a muchos inconvenientes tanto internos como externos este común proceso se ha visto rodeado en deducción o desmejora del mismo, producto de la carencia de valores tanto éticos como morales de sus dirigentes, mermando así la calidad de la educación y dejando de un lado un aspecto tan importante como lo es la complacencia de las necesidades de la población universitaria por falta de una buena gerencia universitaria constituida con principios éticos.

En cuanto a la ética en las organizaciones de educación universitaria, el gerente es esa figura de autoridad con habilidades y capacidades de gerenciar o administrar de la manera más efectiva, eficaz los recursos disponibles dando el mejor uso y destino a estos con la intención de cubrir complacidamente las necesidades presentes en dicha casa de estudio.

Así, se hace impostergable crear estructuras organizativas concretas eficientes, menos burocráticas con personal de excelencia, seleccionados por sus conocimientos, no por amistad, compromisos políticos, así como utilizar intensamente las nuevas tecnologías para dar soporte a los nuevos procesos de gestión, estructuras, equipos que deben ser más próximos y más útiles para la toma de decisiones de la dirección. Entretanto, no olvidemos lo que sobre este tópico nos aporta Aquino, (1999): la gerencia universitaria tiene que

...ser ejercida en base a un conjunto de valores que han sido debidamente identificados y recogidos en el marco legal regulatorio, como son: la identidad y cultura nacional; el respeto al ser humano, su dignidad y su libertad; la libertad de discusión y el pluralismo ideológico, político y religioso; el espíritu democrático, la justicia social y la solidaridad humana; el rigor científico y la responsabilidad ética en la búsqueda y construcción del conocimiento; la creatividad, la criticidad, la integridad y la responsabilidad; la igualdad de oportunidades en el acceso a los beneficios de la educación universitaria (pág. 56). 
Al respecto, Braudel (1958), establece "la gerencia universitaria es una historia que se despliega desde la que es posible vislumbrar, hasta la transferencia y evolución de los principales modelos" (p. 21). En otras palabras, las transformaciones posibles del sistema de educación universitario y de sus universidades están vinculadas a modelos de competencia, que a través de su viabilidad y competitividad permitan su traspaso, composición y ajuste a las condiciones de un entorno económico y social versátil y a los progresivos requerimientos del desarrollo científico y tecnológico.

\subsection{La Gerencia en Contextos de Postmodernidad}

La posmodernidad no significa una ruptura de la corriente modernista, por el contrario, se encuentra relacionado con ésta, por lo que es considerado a juicio de Maestre, (1999) como "(...) un nuevo escenario, como una valoración de lo moderno y no una revolución contra él (...)” (pág. 2).

Por lo que, para el siglo XXI, según Useche y Queipo (2005) exponen "el recurso humano será el actor principal para innovar, crear y será considerado como un punto crucial para el desenvolvimiento organizacional del futuro...". Por lo tanto, las organizaciones en la postmodernidad demandarán una fuerza laboral capaz de crear, facilitar y aplicar conocimiento, sumamente especializado, preparado para desempeñarse en todas las actividades del proceso, como: adquisición, socialización, transformación, estructuración, adición de valor, detección de oportunidades y revisión del conocimiento, estableciendo.

Aunado a esto, las organizaciones en la posmodernidad son entendidas como sistemas que reciben aportaciones del recurso humano, están basadas en la mejora permanente, que según Muñoz y Riverola (2003) "no importa lo superfluo o complejo que pueda ser o hasta lo más transformador, con tal dirección a la organización hacia una transformación, potenciando la capacidad de superación" (pág. 211), o lo que es lo mismo sus actividades ya 
no están fundadas en el imaginario de estándares modernistas; donde lo más importante es la capacidad del capital humano, aprender de éste y adaptarse al mismo, usando sus sapiencias para crear e innovar.

En este momento, existe una diversidad de escritos filosóficos sobre la posmodernidad, para hacer aclaraciones y compilaciones sobre dicha temática; sin embargo, en el ámbito gerencial es poco lo que se ha escrito, de ahí la motivación y significación de este artículo. Donde el desempeño y la direccionalidad organizacional, obligan a adecuar y flexibilizar sus propiedades para afrontar las nuevas circunstancias de caos y complejidad, donde la responsabilidad social y la ética son compromisos elementales en el desenvolvimiento organizacional.

\section{Metodología}

El proceso metodológico, es el camino o recorrido a seguir que alcanzará la investigación, en relación a los Valores Éticos del Gerente Universitario en los Contextos de la Posmodernidad porque se parte del análisis y reflexión crítica de los elementos existentes en el contexto de investigación bibliográfica y documental, lo que constituye la ética del gerente universitario. En consecuencia, se procede a la revisión bibliográfica requerida para desarrollar los aspectos del tema objeto de estudio.

En orden a estas premisas, el estudio se concierne con una investigación de tipo documental, según Ortiz y García, (2002), "la investigación documental es la presentación de un escrito formal que sigue una metodología reconocida consiste primordialmente, en la presentación selectiva de lo que expertos ya han dicho o escrito sobre un tema determinado" (pág. 56). Debido a esto, la cantidad de indagación que se genera en todo el mundo es enorme. No obstante, adquirir estos conocimientos se hace con costumbre mediante la investigación documental realizada en principios 
secundarios. Además, puede presentar la posible conexión de ideas entre varios autores y las ideas del investigador.

\section{Consideraciones Finales}

En este momento del trabajo, se pueden concebir algunas conclusiones a las que se ha llegado, luego de la consulta de las fuentes bibliográficas oportunas al tema con el propósito de obtener una visión más profunda de la temática tratada y aportar razones para que los Valores Éticos se inserten en contextos universitarios posmodernos, por lo tanto, nos conduce a mostrar algunas consideraciones:

Se define la ética como un factor determinante en la actitud de los individuos, que marca la diferencia en el actuar acorde con el ser o el deber ser, dependiendo los criterios valorativos que se tengan al respecto. Partiendo de este supuesto humanista se infiere que el gerente universitario está obligado a actuar éticamente en todo momento y en todo lugar, porque su actuación es ejemplo a seguir por sus subordinados.

Respecto, a los Valores Éticos se pudo concebir son como la guía para resaltar el buen comportamiento humano, para ser exteriorizado a nivel individual y social. Esta actitud ética se transforma en un bien humano que en definitiva, es el fin por el cual se rige la educación, por el cual todos debemos luchar y preservar con miras a la formación de un individuo apegado a la justicia, la equidad, el respeto y la paz.

En relación, a la actitud ética del gerente universitario se puede señalar que el mismo debe poseer sentido de pertenencia y pertinencia con la misión y visión de la organización, desarrollar un espíritu democrático en las acciones a emprender, ser creativo e innovador en la aplicación de estrategias acordes con el contexto en el cual le corresponda desenvolverse y por último tener presente la responsabilidad como fundamento para alcanzar metas propuestas. 
Entretanto, las organizaciones posmodernas registran la importancia que tiene en estos tiempos de cambios la visión del recurso humano como actor creador e innovador y la reinversión organizacional para poder ajustarse y superarse a sí mismas, liberando procesos, productos y servicios, que les permita ser más competitivos.

Sintetizando, el siglo XXI reclama pensamientos rápidos y coherentes a la realidad, para que las respuestas a los cambios sean adecuadas, pertinentes, precisas y sobre todo fructíferas y fuertes, no solo para la organización sino también para su entorno y la sociedad, es decir, la posmodernidad reclama a las organizaciones capacidad de respuestas claras a situaciones circunstanciales.

\section{Referencias}

Aquino, L. (1999). Los Valores como Determinantes de la Conducta Docente. Revista Encuentro Educacional, Vol. 12 Universidad Del Zulia. Venezuela.

Bello, J. (2008). Valores para construir ética. Liven Editores C.A. Venezuela Braudel, I. (1958). Filosofía. Ediciones Insula. Caracas.

Carreras, LI. (2001). Cómo Educar en Valores. México. Ediciones Narcea Correa, M. (2006). Ética Profesional e Identidad Institucional. Universidad Autónoma De Sinaloa. México.

Freites, R. (2012). La Ética en la Construcción Teórica de la Actitud del Gerente Educativo. Volumen 9, Número 1. Instituto Universitario Pedagógico "Monseñor Rafael Arias Blanco" (IUPMA). Maracay, Venezuela. Recuperado de:

http://revistas.upel.edu.ve/index.php/dialogica

George, T. (2009). La Ética y la Gerencia. Un Dilema Ético. [Documento en línea]. Recuperado de:

http://hoteleriadeladdy.blogspot.com/2009/10/un-dilema-etico.html 
González de T., M. (2009). Episteme de la Gerencia Educativa y la Arqueología del Pensamiento Gerencial. [Documento en línea]. Recuperado de:

http://servicio.bc.uc.edu.ve/educacion/revista/n35/art8.pdf

Guédez, V. (2004). La Ética Gerencial. Instrumentos estratégicos que facilitan decisiones correctas. Colombia. Planeta.

Ibáñez, N. y Castillo, R. (2010) Hacia una Epistemología de la Gerencia. Orbis. Revista Científica Ciencias Humanas, vol. 6, núm. 16, pp. 54-78 Fundación Miguel Unamuno y Jugo. Maracaibo, Venezuela. Recuperado de: http://www.redalyc.org/pdf/709/70916426004.pdf Kurosawa, I. (2007). Gerencia. IV edición, Caracas: McGraw-Hill. Maestre, A. (1999). La Modernidad en cuestión: fragmentos de un Debate. Meta política en Revista Trimestral. Vol. 3. No. 11, pp. 1-2.

Muñoz, B. y Riverola J. (2003). Del buen pensar y mejor hacer. Mejora permanente y gestión del conocimiento. Editorial McGraw-Hill. España.

Ortiz, F. y García, M. (2002). Metodología de la Investigación: el Proceso y sus Técnicas. Ed. Limusa.

Paz, Y. (2005). Desarrollo de Competencias para el Comportamiento Ético-Gerencial: Un Enfoque de Responsabilidad. [Monografía]. Cuaderno de Administración, 35.

Ramos, G. (2004) Valores y Autoestima. Valencia, Venezuela: UC.

Rojas, E. (2007). Curso Ética y Docencia. Unidad III: Análisis Deontológico de la Profesión Docente. Universidad Pedagógica Experimental Libertador. Instituto Pedagógico de Barquisimeto “Dr. Luis Beltrán Prieto Figueroa". Departamento de Formación Docente.

Useche, M. y Queipo, B. (2005). Las Organizaciones Posmodernas en el siglo XXI. Mañongo, № 24, pp. 207-216. 


\section{Sonia María Aguilar Rondón}

e-mail: sonia aguilar1965@hotmail.com

Nacida en Valera, estado Trujillo, Venezuela. Actualmente cursa estudios de Doctorado en Ciencias de la Educación en la Universidad Nacional Experimental "Rafael María Baralt". Maestría en Administración de la Educación Básica Magíster Scientiarum 1994-1997; Diplomado en Cooperativismo y Gestión Comunitaria 2007 en el Instituto Universitario de Tecnología del Estado Trujillo; Curso de: Didáctica Especial en el Área de Castellano y Literatura, Matemática y Física y en el Área de Biología y Química 160 Horas 2010 (Diplomado) en el Liceo Bolivariano Antonio José Pacheco Estado Trujillo, convenio Cuba Venezuela. Experiencia Laboral actualmente Prof. Agregado de la Universidad Politécnica Territorial "Mario Briceño Iragorry". 